hep-ph/0009066

IFIC/00-048

\title{
Reconciling neutrino anomalies in a simple four-neutrino scheme with $R$-parity violation
}

\author{
M. Hirsch ${ }^{1}$ and J. W. F. Valle ${ }^{2}$ \\ Instituto de Física Corpuscular - C.S.I.C. - Universitat of València, \\ Edificio Institutos de Paterna, Apartado de Correos 2085 \\ 46071 València, Spain
}

PACS: 12.60Jv, $14.60 \mathrm{Pq}$

\begin{abstract}
We propose a simple extension of the MSSM based on extra compact dimensions which includes an $S U(2) \otimes U(1)$ singlet superfield. The fermion present in this superfield is the sterile neutrino, which combines with one linear combination of $\nu_{e}-\nu_{\mu}-\nu_{\tau}$ to form a Dirac pair whose mass accounts for the LSND anomaly. Its small mass can be ascribed to a volume suppression factor associated with extra compact dimensions. On the other hand the sterile neutrino scalar partner can trigger the spontaneous violation of R-parity, thereby inducing the necessary mass splittings to fit also the solar and atmospheric neutrino data. Thus the model can explain all neutrino oscillation data. It leads to four predictions for the neutrino oscillation parameters and implies that the atmospheric neutrino problem must include at least some $\nu_{\mu} \rightarrow \nu_{s}$ oscillations, which will be testable in the near future. Moreover it also predicts that the lightest supersymmetric particle (LSP) decays visibly via lepton number violating modes, which could be searched for at present and future accelerators.
\end{abstract}

\section{Introduction}

It is well-known that within the standard framework with only three light neutrinos it is impossible to reconcile solar and atmospheric neutrino data [1] with those of LSND [2]. A solution to the atmospheric neutrino problem requires a $\Delta m^{2}$ of the order of $\Delta m^{2} \simeq($ few $) 10^{-3} \mathrm{eV}^{2}[3,4]$, while solar neutrino data could be explained either by $\Delta m^{2} \sim \mathcal{O}\left(10^{-5}\right) e V^{2}$ or $\Delta m^{2} \sim \mathcal{O}\left(10^{-7}\right)$

$\overline{1}$ (a) mahirsch@neutrinos.uv.es

2 (a) valle@neutrinos.uv.es

Preprint submitted to Elsevier Preprint

4 November 2018 
$\mathrm{eV}^{2}[3,5]$ (LMA or LOW solutions of the solar neutrino problem), none of which can be reconciled with the scale $\Delta m^{2} \sim 0.3-1 \mathrm{eV}^{2}$ indicated by the LSND experiment [2]. Since the LSND experiment has not been confirmed independently, many theoretical papers have chosen to ignore this result ${ }^{3}$.

A simple way to account for all neutrino data would be to postulate the existence of an additional neutrino state $[7,8]$, which due to the constraints from LEP has to be mainly sterile.

However, even including a sterile neutrino, there are essentially only two neutrino spectra allowed by the data. One of the possibilities is shown in Fig. (1). It consists of two nearly degenerate pairs of neutrinos separated by a gap of the order of the LSND scale. The two pairs then have to be split by $\Delta m_{i j}^{2}$ which correctly fit solar and atmospheric data. For recent analyses of atmospheric and solar neutrino data in four-neutrino models see ref. [9,10]. In Fig. (1) we show only the case where the lower $\Delta m_{i j}^{2}$ corresponds to the solar scale. A second spectrum with $\Delta m_{\text {atm }}^{2} \leftrightarrow \Delta m_{\text {sol }}^{2}$ is equally well allowed by the data $\square$.

The question then is: Can one make sense of such a neutrino spectrum theoretically?

A number of attempts can be found in the literature [11]. While originally motivated by the desire to account for hot dark matter [12-14] soon after LSND results came into existence, it was realized that schemes leading to these spectra would easily fit LSND results together with solar and atmospheric data.

Here we propose, what we believe to be one of the simplest particle physics model for the inclusion of a sterile neutrino state into the spectrum. It is based on a minimal extension of the MSSM with one additional singlet superfield field. The fermionic component of the singlet combines with (one of) the active neutrinos to form a light Dirac state at the LSND scale. Its scalar neutrino component develops a nonzero vacuum expectation value (vev) $[15,16]$, breaking R-parity spontaneously [17] and effectively generating bilinear R-parity violating terms in the superpotential $[18,19]$.

Supersymmetry with bilinear R-parity violation has been shown to provide a predictive theory for solar and atmospheric neutrino oscillations in which the neutrino masses and mixing angles are all determined in terms of the three fundamental bilinear terms [20,21]. In the context of the present 4-neutrino scheme the breaking of R-parity leads to Majorana masses for the neutrinos, splitting the Dirac neutrino into a quasi-Dirac pair [22], and giving mass to one additional neutrino state. It is this breaking of R-parity that leads to the solar and atmospheric oscillations. The model has therefore, despite being minimal-

3 The KARMEN experiment [6] does rule out some parts of the parameter space favoured by LSND. However, it does not disprove LSND.

4 The case where $\Delta m_{\text {sol }}^{2}$ and $\Delta m_{a t m}^{2}$ are exchanged is allowed, but can not be realized in our model. 


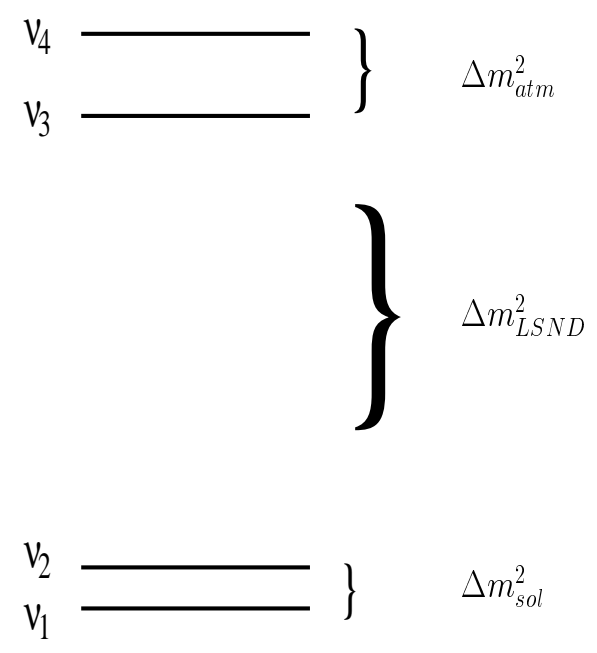

Fig. 1. Four-neutrino spectrum fitting solar, atmospheric and LSND data.

istic, all the basic ingredients for solving solar and atmospheric neutrinos in addition to the LSND data. Moreover it extends the predictivity of the bilinear $\not R_{p}$ model to the 4-neutrino case: four parameters are predictable, out of a total of ten ( 4 masses and six mixing angles) Ð. In contrast with the early models, here the smallness of the LSND scale arises without appealing to a radiative mechanism, but due to the volume factor associated with the canonical normalization of the wave-function of the bulk field in the compactified dimensions, as suggested in ref. [23].

This paper is organized as follows. In the next section we describe the main features of the model. Then, we discuss some numerical results, exploring what are the parameter ranges which could fit the neutrino data. We also discuss, how this model can be tested by both, future neutrino measurements as well as at accelerators, before giving a short conclusion.

\section{The model}

The model we advocate can be regarded as a simple extension of the bilinear R-parity broken MSSM [18,19]. The minimal extension beyond the MSSM particle content is to include a single right-handed neutrino. Thus to the su-

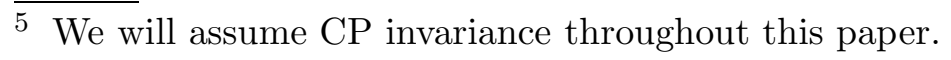


perpotential of the MSSM we add,

$$
W=W_{M S S M}+h_{i}^{\nu} \widehat{L}_{i} \widehat{H}_{u} \widehat{\nu}^{c}
$$

The last term in the equation above gives three Dirac mass terms

$$
d_{i}=h_{i}^{\nu} v_{u}
$$

giving automatically a Dirac mass to one linear combination of $\nu_{e}-\nu_{\mu}-\nu_{\tau}$ once $H_{u}$ develops a nonzero vev. The size of this entry is governed by the magnitude of the corresponding Yukawa couplings $h_{i}^{\nu}$.

However, within a supersymmetric framework the $h_{i}^{\nu} \widehat{L}_{i} \widehat{H}_{u} \widehat{\nu}^{c}$ term can have also other consequences.

In SUSY models the scalar partner in the $\nu^{c}$ superfield can, depending on the parameters of the model, acquire a vacuum expectation value $\left(v_{R}\right)$ through the usual Higgs mechanism, breaking R-parity spontaneously, as proposed in ref. [16]. For this all we need is to have its squared mass negative at the weak scale, like in the standard model Higgs mechanism. First note that this may happen as a result of the primordial choice of non-universal boundary conditions at the unification scale which make $m_{\nu^{c}}^{2}$ different from the $S U(2) \otimes U(1)$ non-singlet soft scalar masses. Alternatively it might, under some circumstances, be induced dynamically via radiative corrections [24].

Such a right-handed sneutrino vev, however, produces effectively bilinear terms in the superpotential via

$$
\epsilon_{i}=h_{i}^{\nu} v_{R}
$$

It is well-known that the existence of such lepton number violating (R-parity breaking) bilinears lead automatically to Majorana masses for the neutrinos.

In the following we will assume a non-zero $v_{R}$. With this one can immediately write down the mass matrix of the model. Choosing as basis $\Psi_{0}^{\prime T}=$ $\left(\nu_{e}, \nu_{\mu}, \nu_{\tau}, \nu^{c},-i \lambda^{\prime},-i \lambda_{3}, \psi_{H_{d}}^{1}, \psi_{H_{u}}^{2}\right)$ we find that the neutrino-neutralino mass matrix of this model is given by:

$$
\mathcal{M}_{0}^{8}=\left(\begin{array}{cc}
m_{D} & m_{\mathbb{R}_{p}} \\
m_{\mathbb{R}_{p}}^{T} & \mathcal{M}_{\chi^{0}}
\end{array}\right) .
$$


where $\mathcal{M}_{\chi^{0}}$ is the MSSM neutralino mass matrix,

$$
\mathcal{M}_{\chi^{0}}=\left(\begin{array}{cccc}
M_{1} & 0 & -\frac{1}{2} g^{\prime} v_{d} & \frac{1}{2} g^{\prime} v_{u} \\
0 & M_{2} & \frac{1}{2} g v_{d} & -\frac{1}{2} g v_{u} \\
-\frac{1}{2} g^{\prime} v_{d} & \frac{1}{2} g v_{d} & 0 & -\mu \\
\frac{1}{2} g^{\prime} v_{u} & -\frac{1}{2} g v_{u} & -\mu & 0
\end{array}\right)
$$

and the Dirac mass matrix $m_{D}$ is given by,

$$
m_{D}=\left(\begin{array}{cccc}
0 & 0 & 0 & \frac{1}{\sqrt{2}} h_{1} v_{u} \\
0 & 0 & 0 & \frac{1}{\sqrt{2}} h_{2} v_{u} \\
0 & 0 & 0 & \frac{1}{\sqrt{2}} h_{3} v_{u} \\
\frac{1}{\sqrt{2}} h_{1} v_{u} \frac{1}{\sqrt{2}} h_{2} v_{u} & \frac{1}{\sqrt{2}} h_{3} v_{u} & 0
\end{array}\right)
$$

Finally, $m_{\mathbb{R}_{p}}$ contains the entries induced by the breaking of R-parity. These can be read off from the mass matrix of the spontaneous model as,

$$
m_{R_{p}}=\left(\begin{array}{cccc}
-\frac{1}{2} g^{\prime}\left\langle\tilde{\nu}_{e}\right\rangle & \frac{1}{2} g\left\langle\tilde{\nu}_{e}\right\rangle & 0 & -\epsilon_{e} \\
-\frac{1}{2} g^{\prime}\left\langle\tilde{\nu}_{\mu}\right\rangle & \frac{1}{2} g\left\langle\tilde{\nu}_{\mu}\right\rangle & 0 & -\epsilon_{\mu} \\
-\frac{1}{2} g^{\prime}\left\langle\tilde{\nu}_{\tau}\right\rangle & \frac{1}{2} g\left\langle\tilde{\nu}_{\tau}\right\rangle & 0 & -\epsilon_{\tau} \\
0 & 0 & 0 \sum h_{i}^{\nu}\left\langle\tilde{\nu}_{i}\right\rangle
\end{array}\right) .
$$

Here $\left\langle\tilde{\nu}_{i}\right\rangle$ are the left-handed sneutrino vevs, which are in general non-zero due to the minimization conditions of the scalar potential once we have nonzero $\epsilon_{i}$. Note that both $m_{\mathbb{R}_{p}}$ and $m_{D}$ have such a structure that with either term present only one neutrino would gain a mass. Only if $m_{D}$ and $m_{R_{p}}$ are not completely "aligned" to each other (i.e. if at least one of the ratios $h_{i} / h_{j}$ differs from $\Lambda_{i} / \Lambda_{j}$ where $\left.\Lambda_{i}=\epsilon_{i} v_{d}+\mu\left\langle\tilde{\nu}_{i}\right\rangle\right)$ we have three non-zero masses plus one massless state in the spectrum.

Depending on the relative size of $|\Lambda|^{2} / M_{S U S Y}^{4}$ and $\left|h^{\nu}\right|$, where,

$$
\begin{gathered}
|\Lambda|=\sqrt{\Lambda_{e}^{2}+\Lambda_{\mu}^{2}+\Lambda_{\tau}^{2}} \\
\left|h^{\nu}\right|=\sqrt{\left(h_{1}^{\nu}\right)^{2}+\left(h_{2}^{\nu}\right)^{2}+\left(h_{3}^{\nu}\right)^{2}}
\end{gathered}
$$

the heaviest state will be either a quasi-Dirac pair or a Majorana neutrino. Possible parameter choices which fit the neutrino oscillation data are discussed in the next section. 
Before closing this section let us comment on the required smallness of $h_{i}^{\nu}$. One way to avoid having to simply postulate as a phenomenological assumption is to assume the existence of extra compact dimensions, probed only by gravity and possibly gauge-singlet fields, which can lower the fundamental scales to the weak scale (TeV) [25]. There have been many recent papers applying the idea of extra dimensions to neutrino physics [26]. Using the Gauss law one can write

$$
M_{P l}^{2} \simeq\left(R M_{F}\right)^{n} M_{F}^{2}
$$

where $R$ is the compactification radius of the additional dimensions and $M_{F}$ is the fundamental Planck scale, which in these theories can be low. A recent attempt in this direction has been presented in ref. [23]. It postulates that $M_{F} \simeq 10 \mathrm{TeV}$ and a $4+\mathrm{n}$ dimensional theory with $n=6$ for which the corresponding value of $R$ is $R \simeq 10^{-12} \mathrm{~cm}$. This way the smallness of $h_{i}^{\nu}$ (and hence of the LSND mass scale) will follow from the volume factor associated with the canonical normalization of the wave-function of the bulk field in the compactified dimensions. Our fourth light neutrino $\nu_{s}$ ( $s$ for sterile) is identified with the zero mode of the Kaluza-Klein states. To first approximation the sterile neutrino combines with a combination of the active neutrinos (mainly

$\nu_{\mu}$ with some $\nu_{\tau}$ ) in order to form a Dirac neutrino with mass in the eV range leaving the other two neutrinos massless. Thus we can apply exactly the same construction in the present case. In other words, the present model may be regarded as a variant of that in ref. [23] in which neutrino mass splittings are now due to the breaking of R-parity. As we discuss below this has important phenomenological advantages.

\section{Numerical results}

We now turn to the pattern of neutrino masses and mixings arising from our model. Starting with the masses we show in Fig. (2) the eigenvalues which follow from diagonalization of the mass matrix for a specific, though arbitrary choice of parameters as a function of $|\Lambda|$. For small values of $|\Lambda|$ two neutrino states form a Dirac pair. Increasing the size of the R-parity breaking (while keeping other parameters fixed) increases the mass splitting within this pair, as well as the mass of the second mass eigenstate lying at the solar neutrino scale. In this example, for $|\Lambda| \simeq 0.05 \mathrm{GeV}^{2}$ the three mass squared differences are of the right order of magnitude for solving the neutrino problems. Note, that the actual values of the MSSM parameters are not essential. Larger or smaller values of MSSM masses could be accounted for by a simple appropriate rescaling of $|\Lambda|$.

One sees from Fig. (2) that for small values of $|\Lambda| \nu_{3}$ and $\nu_{4}$ combine to form a quasi-Dirac pair. The splitting between those two states increases with increasing $|\Lambda|$. 

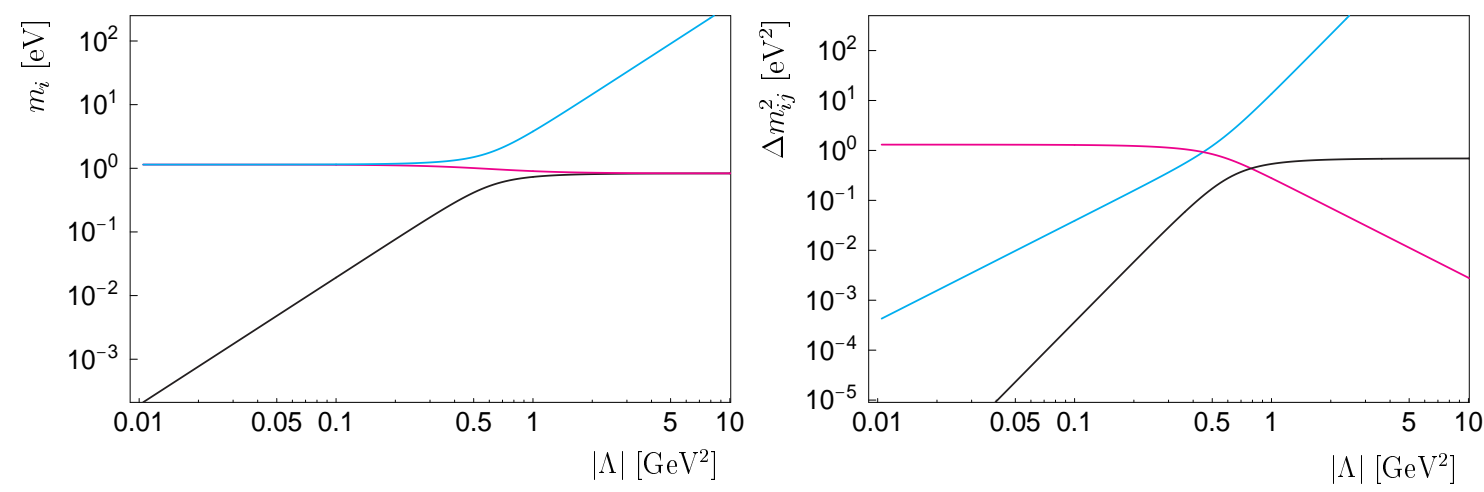

Fig. 2. Example of calculated mass eigenvalues and corresponding mass squared differences as a function of $|\Lambda|$ for an arbitrary but fixed choice of other model parameters. To the left, mass eigenvalues. To the right, $\Delta m_{42}^{2}, \Delta m_{43}^{2}$ and $\Delta m_{21}^{2}$. Parameters which have been kept fixed for the calculation shown in this plot are $\left|h^{\nu}\right|=5 \times 10^{-12}, \mu=500 \mathrm{GeV}, M_{2}=200 \mathrm{GeV}$ and $\tan \beta=2.5$.

Turning now to mixings, a general weak interaction four-neutrino model requires, in addition to the four masses, six mixing angles (and six CP phases if $\mathrm{CP}$ is violated) in order to characterize the structure of the weak leptonic current [8].

As explained above the present model has a high degree of predictivity, since now all 10 neutrino oscillation parameters are given in terms of six independent quantities which may be conveniently chosen as the $d_{i}$ and the alignment parameters $\Lambda_{i}$. Due to the small left-handed sneutrino vevs induced by the minimization of the scalar potential the $\Lambda_{i}$ ratios are not proportional to the $h_{i}$ ratios, thereby breaking the projectivity of the neutrino mass matrix. This is crucial to induce the solar neutrino oscillations.

Within any model producing a $(2,2)$-neutrino spectrum as shown in Fig. (1) the conversion probabilities relevant for reactor, atmospheric and LSND experiments are modified with respect to the usual 2-generation formula. ${ }^{6}$ In order to compare the experimental data with the results of our model we therefore give in the following the conversion (and survival) probabilities corresponding to the neutrino spectrum of Fig. (1).

For the reactor neutrinos one finds after some trivial algebraic manipulations,

$$
P_{\nu_{e} \rightarrow \nu_{e}}^{C H O O Z}=1-4 \sin ^{2}\left(\Delta m_{L S N D}^{2} \frac{L}{E}\right)\left|\left(1-U_{e 3}^{2}-U_{e 4}^{2}\right)\left(U_{e 3}^{2}+U_{e 4}^{2}\right)\right|
$$

6 Also the solar neutrino survival probability will receive a modification. However, $\mathrm{CHOOZ}$ and atmospheric neutrino results imply that this correction is negligible. 

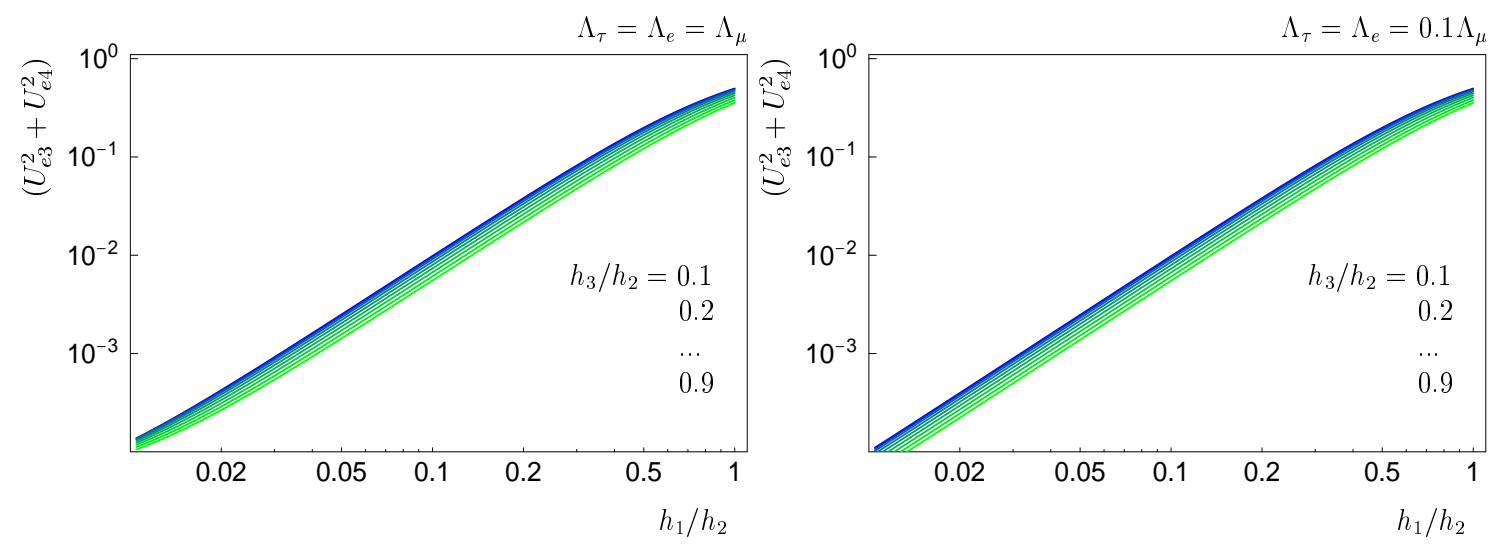

Fig. 3. Two examples of $\left(U_{e 3}^{2}+U_{e 4}^{2}\right)$ as a function of $h_{1} / h_{2}$ for different choices of $h_{3} / h_{2}$. This quantity is constrained by CHOOZ [27] and implies $h_{1}<h_{2}$. Only a rather weak dependence on other model parameters is found.

$$
-4 \sin ^{2}\left(\Delta m_{a t m}^{2} \frac{L}{E}\right)\left|U_{e 3}\right|^{2}\left|U_{e 4}\right|^{2}
$$

In Fig. (3) we show two examples of $\left(U_{e 3}^{2}+U_{e 4}^{2}\right)$ as a function of $h_{1} / h_{2}$ for different choices of $h_{3} / h_{2}$. This quantity is constrained by the non-observation of oscillations at reactor experiments and has to be rather small, implying that $h_{1}<h_{2}$.

Similarly for the LSND oscillation probability one finds,

$$
\begin{aligned}
P_{\bar{\nu}_{\mu} \rightarrow \bar{\nu}_{e}}^{L S N D} & =4 \sin ^{2}\left(\Delta m_{L S N D}^{2} \frac{L}{E}\right)\left|\left(U_{e 1} U_{\mu 1}+U_{e 2} U_{\mu 2}\right)\left(U_{e 3} U_{\mu 3}+U_{e 4} U_{\mu 4}\right)\right| \\
& =: \sin ^{2}\left(\Delta m_{L S N D}^{2} \frac{L}{E}\right) \sin ^{2}\left(2 \theta_{L S N D, \text { eff }}\right)
\end{aligned}
$$

Since LSND is sensitive only to the largest mass gap in the spectrum, this corresponds simply to a re-interpretation of the effective mixing angle. In Fig. (4) we show $\sin ^{2}\left(2 \theta_{L S N D, \text { eff }}\right)$ as a function of $h_{1} / h_{2}$ for different values of $h_{3} / h_{2}$. For $\sin ^{2}\left(2 \theta_{L S N D, \text { eff }}\right) \sim 10^{-2}-10^{-3}, h_{1} / h_{2} \sim 0.02-0.1$ is needed.

Slightly more complicated is the conversion probability for the atmospheric neutrinos, given as

$$
\begin{aligned}
P_{\nu_{\mu} \rightarrow \nu_{x}}^{a t m} & =P_{\nu_{\mu} \rightarrow \nu_{\tau}}^{a t m}+P_{\nu_{\mu} \rightarrow \nu_{s}}^{a t m} \\
& =4 \sin ^{2}\left(\Delta m_{L S N D}^{2} \frac{L}{E}\right)\left\{\left|\left(U_{\mu 1} U_{\tau 1}+U_{\mu 2} U_{\tau 2}\right)\left(U_{\mu 3} U_{\tau 3}+U_{\mu 4} U_{\tau 4}\right)\right|+|\tau \rightarrow \mathrm{s}|\right\} \\
& +4 \sin ^{2}\left(\Delta m_{a t m}^{2} \frac{L}{E}\right)\left\{\left|U_{\mu 3} U_{\tau 3} U_{\mu 4} U_{\tau 4}\right|+|\tau \rightarrow \mathrm{s}|\right\}
\end{aligned}
$$



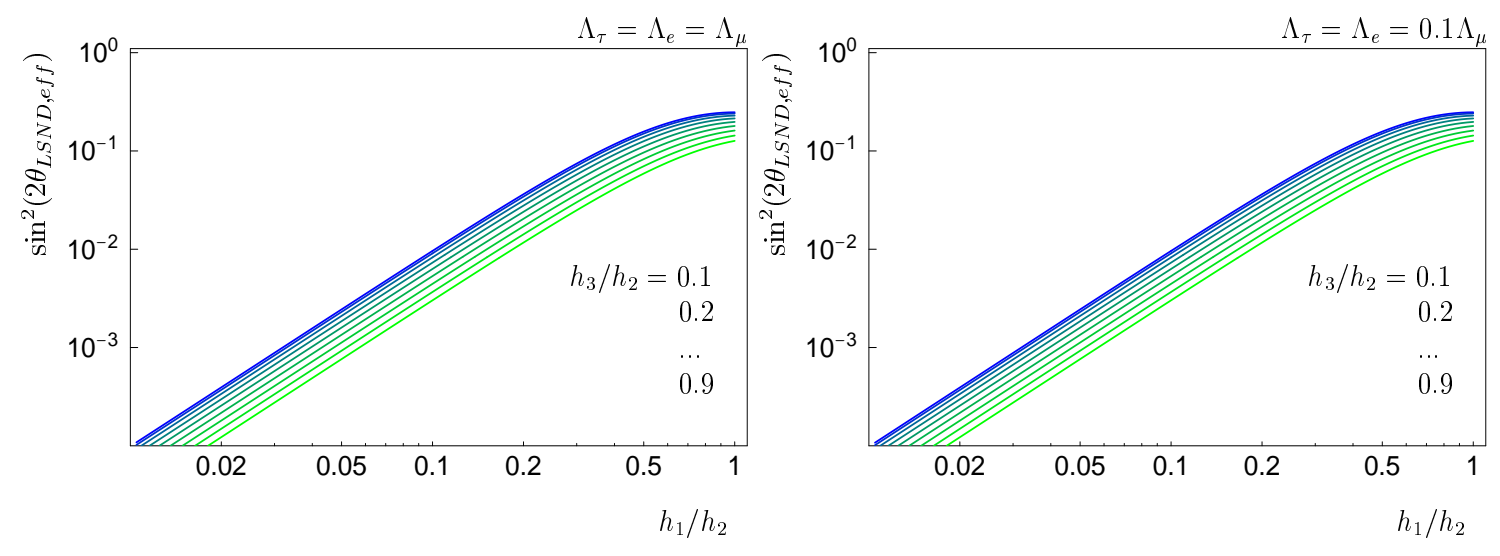

Fig. 4. The "effective" LSND angle for the neutrino spectrum of Fig. (1) as a function of $h_{1} / h_{2}$ for different choices of $h_{3} / h_{2}$. Obviously to explain the LSND result $h_{1} \ll h_{2}$ is required.

The first term proportional to $\sin ^{2}\left(\Delta m_{L S N D}^{2} \frac{L}{E}\right)$ will be averaged over in the atmospheric neutrino data (except for the smallest $L$ and largest $E$ ) and should be visible in the data as a finite "offset" proportional to $2\left\{\mid\left(U_{\mu 1} U_{\tau 1}+\right.\right.$ $\left.\left.U_{\mu 2} U_{\tau 2}\right)\left(U_{\mu 3} U_{\tau 3}+U_{\mu 4} U_{\tau 4}\right)|+| \tau \rightarrow \mathrm{s} \mid\right\}$. The term $4\left\{\left|U_{\mu 3} U_{\tau 3} U_{\mu 4} U_{\tau 4}\right|+|\tau \rightarrow \mathrm{s}|\right\}$ defines the effective atmospheric neutrino mixing angle in our model.

In Fig. (5) we give the "effective" atmospheric neutrino angle (left) and the corresponding "offset" (right) as a function of $h_{3} / h_{2}$ for different choices of $h_{1} / h_{2}$. The size of this offset depends mainly on $h_{3} / h_{2}$. Note that both reactor and LSND data require $h_{1} \ll h_{2}$.

Note that in the (unrealistically) extreme case of $h_{1}, h_{3} \rightarrow 0$ we would have pure (two generation) $\nu_{\mu} \rightarrow \nu_{s}$ oscillations in our model. However, relatively large values of $h_{3}$ are not excluded by the data, implying that the atmospheric neutrino oscillations in our model are described in general by a mixture of $\nu_{\mu} \rightarrow \nu_{s}$ and $\nu_{\mu} \rightarrow \nu_{\tau}$. Note also that pure $\nu_{\mu} \rightarrow \nu_{\tau}$ oscillations are not allowed in our model, since the quasi-Dirac pair between which the oscillation takes place necessarily involves the sterile state. We have estimated that at least 50 $\%$ of the oscillation probability must be due to $\nu_{\mu} \rightarrow \nu_{s}$ in our model.

It has been stated that present atmospheric neutrino data rule out a pure $\nu_{\mu} \rightarrow \nu_{s}$ conversion [1]. However, we find it premature to confidently rule out this possibility at this stage. Indeed, from a global fit in which uncertainties are treated conservatively [4], this exclusion does not yet emerge, although the pure sterile conversion is indeed disfavored. However, in four-neutrino models like the present, the atmospheric conversions are certainly not pure $\nu_{\mu} \rightarrow \nu_{s}$ in general, as seen from eq. (13). As a result, even if ones rules out pure $\nu_{\mu} \rightarrow \nu_{s}$ conversion one does not rule out the model itself. However it is definitely clear that it will be tested by more refined data yet to come. The bound on the admixture of $\nu_{\mu} \rightarrow \nu_{s}$ can be inferred from the atmospheric data as suggested 

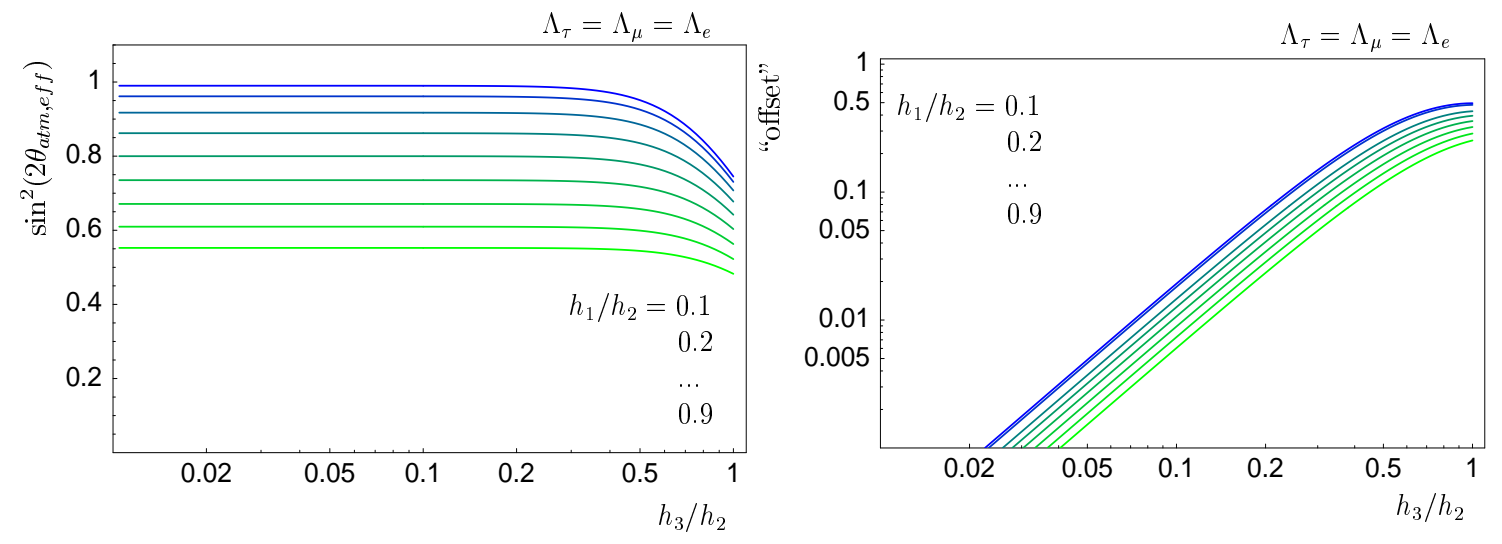

Fig. 5. The "effective" atmospheric neutrino angle (left) and "offset" (right) as a function of $h_{3} / h_{2}$ for different $h_{1} / h_{2}$ choices.
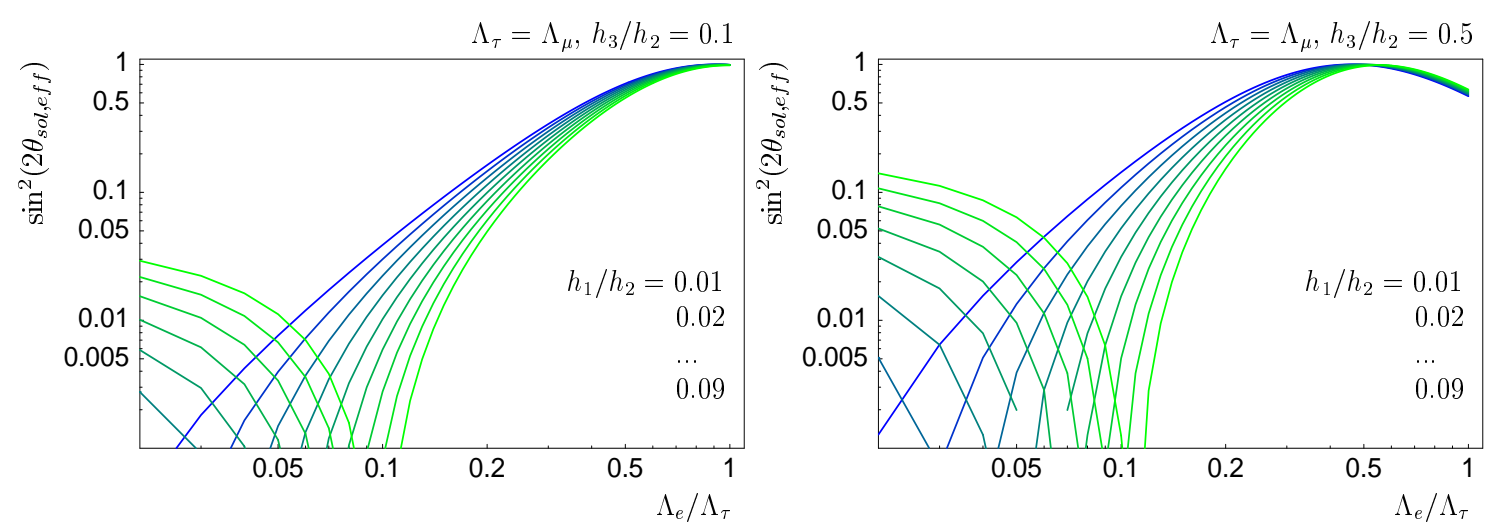

Fig. 6. The "effective" solar angle as a function of $\Lambda_{e} / \Lambda_{\tau}$ for two different choices of $h_{3} / h_{2}$.

in [9]. Moreover, the "offset" predicted to exist in our model, if the atmospheric oscillations are not pure $\nu_{\mu} \rightarrow \nu_{s}$, might be testable by the K2K experiment.

To close the discussion on neutrino angles, in Fig. (6) we display the effective solar angle as a function of $\Lambda_{e} / \Lambda_{\tau}$ for two different choices of other parameters. Due to the flatness of the recoil electron neutrino spectrum indicated by the most recent solar neutrino data [1], the solutions to the solar neutrino problem which are now preferred by the data involve large mixing [3,5]. Large neutrino mixing angles require $\Lambda_{e} \simeq \Lambda_{\tau}$ within a factor of $2-3$. 


\section{Neutralino decay}

Let us finally briefly discuss the decay of the lightest of the 4 heavy states in our neutrino-neutralino spectrum. We will call it for simplicity the lightest neutralino. As is well-known with the presence of the bilinear (lepton number violating) terms in the superpotential the lightest neutralino is no longer stable $[28,29]$ and decays through lepton number violating modes. In the bilinear Rparity violating model it has been shown that this decay occurs with sizeable branching ratio into visible states [20] inside typical detector sizes despite the small neutrino masses required by current neutrino data. Moreover, these decays might be used to obtain information on neutrino angles [30].

Since in our present model R-parity violation is required in order to provide the solar and atmospheric neutrino mass splittings, the neutralino will decay as well. However, two features distinguish the current model from the simpler bilinear model. First, the spontaneous violation of R-parity (which is used as a seed for the generation of the bilinear terms) implies the existence of a Majoron. The neutralino then also can decay via the invisible mode $\chi^{0} \rightarrow J \nu[28]$, see Fig. (7).

However, due to the smallness of the (Dirac) neutrino coupling this decay mode will be very much suppressed when compared to the visible $R_{p}$ violating decays. A very crude order-of-magnitude estimate for the two graphs shown in Fig. (7) gives for the neutral current graph

$$
\Gamma^{v i s} \sim \frac{g^{2}}{16 \pi}\left(\frac{|\Lambda|}{M_{W}^{2}}\right)^{2} m_{\chi^{0}} \sim 10^{-16} \mathrm{GeV}
$$

while for the Majoron decay one expects:

$$
\Gamma^{\text {invis }} \sim \frac{\left|h^{\nu}\right|^{2}}{16 \pi} m_{\chi^{0}} \sim 10^{-22} \mathrm{GeV}
$$

Thus, even though a Majoron exists in the present model, sufficiently large branching ratios to visible states in the decay of $\chi^{0}$ exist to be searched for at accelerators.

Second and more important, however, in the present model the solar neutrino problem is solved by $\nu_{e} \rightarrow \nu_{\tau}$ oscillations (with possibly some admixture of $\nu_{e} \rightarrow \nu_{\mu}$ ). If the solar neutrinos indeed require large mixing, as currently favoured by the data, $\Lambda_{e} \simeq \Lambda_{\tau}$ is needed in our model to fit the data. This would then lead to the prediction that in the decays of the neutralino to semi-leptonic final states a comparable number of electrons and taus should exist. This prediction might be used to distinguish the present model from the bilinear R-parity violating model, since the latter requires that electrons in the semi-leptonic states should be very much suppressed $[20,30]$ reflecting the fact that in the bilinear model $\Lambda_{e} \ll \Lambda_{\mu} \simeq \Lambda_{\tau}$ is required. 

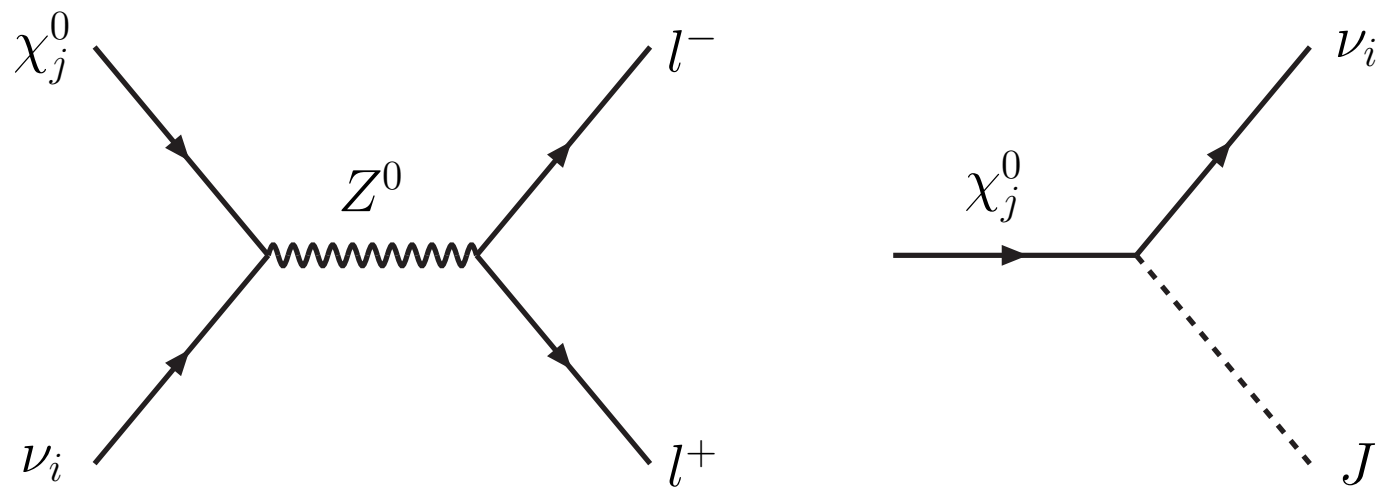

Fig. 7. Two examples of Feynman graphs leading to the decay of the lightest neutralino in our model. To the left, neutral current interaction leading to visible final states. To the right, invisible decay into a Majoron and a neutrino.

\section{Conclusion}

We have discussed a very economical model of neutrino mass which, despite its simplicity is in principle able to fit all neutrino data including LSND. The model is minimal in the sense that we only introduce one additional $S U(2) \otimes U(1)$ singlet superfield into the MSSM superpotential. The LSND scale can be explained by the fermion present in this superfield, while its scalar partner can trigger the spontaneous violation of R-parity, through a nonzero right-handed sneutrino vacuum expectation value, which in turn splits the Dirac neutrino into a quasi-Dirac pair (atmospheric neutrino scale), providing also mass to one of the low-lying neutrinos at the solar neutrino scale. The smallness of the overall neutrino mass (LSND scale) can be ascribed to the volume suppression factor associated with the compactified dimensions. Thus the model can naturally explain all neutrino oscillation data, making four predictions for the neutrino oscillation parameters. We stress that this model is testable in the near future: one of its necessary ingredients is that atmospheric neutrino conversions must include at least some $\nu_{\mu} \rightarrow \nu_{s}$ oscillations. Thus future atmospheric neutrino measurements will provide a crucial test of this model. However, if tides turn, and sterile neutrinos in the future are not disfavored by Superkamiokande, another test of the model could be done at accelerators. The model predicts that the lightest neutralino decays inside the detector with sizeable branching ratios into visible states. 


\section{Acknowledgement}

This work was supported by DGICYT grant PB98-0693 and by the TMR contract ERBFMRX-CT96-0090. M.H. acknowledges support from the European Union's Marie-Curie program under grant No ERBFMBICT983000.

\section{References}

[1] Talks by Toshiyuki Toshito, Yasuo Takeuchi and Koichiro Nishikawa at the XXXth International Conference on High Energy Physics July 27 - August 2, 2000, Osaka, Japan

[2] LSND Collaboration, C. Athanassopoulos et al., Phys.Rev.Lett. 75 (1995) 2650; Phys.Rev.Lett. 77 (1996) 3082; Phys.Rev.Lett. 81 (1998) 1774

[3] An updated discussion of solar and atmospheric neutrino data was presented by M. C. Gonzalez-Garcia at the International Conference on High Energy Physics, Osaka, Japan. Postcript files avalaible from http://neutrinos.uv.es//. For details of the analysis see $[4,5]$.

[4] N. Fornengo, M. C. Gonzalez-Garcia and J. W. F. Valle, Nucl. Phys. B580 (2000) 58 hep-ph/0002147; M.C. Gonzalez-Garcia, et al Nucl. Phys. B543, 3 (1999) and Phys. Rev. D58 (1998) 033004 hep-ph/9801368 and references therein.

[5] M.C. Gonzalez-Garcia, P.C. de Holanda, C. Peña-Garay and J. W. F. Valle, Nucl. Phys. B573, 3 (2000) hep-ph/9906469 and references therein.

[6] K. Eitel, "Statistical analysis of the LSND evidence and the KARMEN exclusion for anti-nu/mu -i, anti-nu/e oscillations," CERN-OPEN-2000-209 In *Geneva 2000, Confidence limits* 173-185.

[7] J. Schechter and J. W. F. Valle, Phys. Rev. D21, 309 (1980).

[8] J. Schechter and J. W. F. Valle, Phys. Rev. D22, 2227 (1980).

[9] O. Yasuda, hep-ph/0006319, and O. Yasuda, talk at the XXXth International Conference on High Energy Physics July 27 - August 2, 2000, Osaka, Japan

[10] C. Giunti, M. C. Gonzalez-Garcia and C. Pena-Garay, Phys. Rev. D62 (2000) 013005 hep-ph/0001101.

[11] For a review see J. W. F. Valle, "Neutrino masses: From fantasy to facts," Springer tracts in modern physics 163 (2000) 35-68, hep-ph/9906378 and references therein. For a recent review on phenomenology of neutrino oscillations see, S.M. Bilenky, C. Giunti and W. Grimus, Prog. Part. Nucl. Phys. 43 (1999) 11

[12] J. T. Peltoniemi, D. Tommasini and J. W. F. Valle, Phys. Lett. B298 (1993) 383.

[13] J. T. Peltoniemi and J. W. F. Valle, Nucl. Phys. B406 (1993) 409 hepph/9302316]. 
[14] D. O. Caldwell and R. N. Mohapatra, Phys. Rev. D48 (1993) 329.

[15] G. G. Ross and J. W. F. Valle, Phys. Lett. B151, 375 (1985); J. Ellis, G. Gelmini, C. Jarlskog, G. G. Ross and J. W. F. Valle, Phys. Lett. B150, 142 (1985); C. S. Aulakh and R. N. Mohapatra, Phys. Lett. B121, 14 (1983); A. Santamaria and J. W. F. Valle, Phys. Lett. B195 (1987) 423; Phys. Rev. D39 (1989) 1780 and Phys. Rev. Lett. 60 (1988) 397.

[16] A. Masiero and J. W. F. Valle, Phys. Lett. B 251 (1990) 273; J.C. Romão, C.A. Santos, J. W. F. Valle, Phys. Lett. B 288 (1992) 311

[17] For a review see J.C. Romão, proceedings of International Workshop on Physics Beyond the Standard Model: From Theory to Experiment, Valencia, 13-17 Oct 1997, Ed. V. Berezinsy, G. Raffelt, J. W. F. Valle, Nuclear Physics B, Proceedings Supplements, 2000,Vol. 81, ISSN 0920-5632

[18] M.A. Díaz, J.C. Romão and J. W. F. Valle, Nucl. Phys. B524, 23 (1998) hepph/9706315]. For reviews see, e.g. J. W. F. Valle, "Super-gravity unification with bilinear R-parity violation," Proceedings of 6th International Symposium on Particles, Strings and Cosmology (PASCOS 98), Boston, MA, 22-27 Mar 1998 hep-ph/9808292 ed. P. Nath; M.A. Díaz, talk given at International Europhysics Conference on High Energy Physics, Jerusalem, Israel, 19-26 Aug 1997, hep-ph 9712213

[19] For some recent references on bilinear R-parity violation, see F. Takayama and M. Yamaguchi, Phys. Lett. B476, 116 (2000) hep-ph/9910320; D. E. Kaplan and A. E. Nelson, JHEP 0001, 033 (2000); E. J. Chun and S. K. Kang, Phys. Rev. D61, 075012 (2000); A. Datta, B. Mukhopadhyaya and S. Roy, Phys. Rev. D61, 055006 (2000); A. S. Joshipura and S. K. Vempati, Phys. Rev. D60, 111303 (1999); E.J. Chun, S. K. Kang, C. W. Kim and U. W. Lee, Nucl. Phys. B544, 89 (1999) hep-ph/9807327]; E. Nardi, Phys. Rev. D55, 5772 (1997) hep-ph/9610540; F. M. Borzumati, Y. Grossman, E. Nardi and Y. Nir, Phys. Lett. B384, 123 (1996) hep-ph/9606251; F. de Campos, M.A. García-Jareño, A.S. Joshipura, J. Rosiek, and J. W. F. Valle, Nucl. Phys. B 451 (1995) 3; T. Banks, Y. Grossman, E. Nardi, and Y. Nir, Phys. Rev. D 52 (1995) 5319; A. S. Joshipura and M.Nowakowski, Phys. Rev. D 51 (1995) 2421; H. P. Nilles and N. Polonsky, Nucl. Phys. B 484 (1997) 333; B. de Carlos, P. L. White, Phys. Rev. D 55 (1997) 4222; S. Roy and B. Mukhopadhyaya, Phys. Rev. D 55 (1997) 7020

[20] M. Hirsch, M. A. Diaz, W. Porod, J. C. Romao and J. W. F. Valle, hepph/0004115, to appear in Phys. Rev. D

J. C. Romão, M. A. Diaz, M. Hirsch, W. Porod and J. W. F. Valle, Phys. Rev. D61, 071703 (2000) hep-ph/9907499

[21] For papers discussing R-parity violation phenomenology see, e.g. F. de Campos, O. J. Eboli, M. A. Garcia-Jareno and J. W. F. Valle, Nucl. Phys. B546, 33 (1999) hep-ph/9710545]; M. Nowakowski and A. Pilaftsis, Nucl. Phys. B461, 19 (1996) hep-ph/9508271]; L. Navarro, W. Porod and J. W. F. Valle, Phys. Lett. B459, 615 (1999) hep-ph/9903474]; M. A. Diaz, D. A. Restrepo and J. W. F. Valle, hep-ph/9908286 [to be published in Nucl. Phys. B]; B. Allanach et al., hep-ph/9906224; A. Faessler, S. Kovalenko and F. Simkovic, Phys. Rev. D58, 055004 (1998) hep-ph/9712535; S. Davidson, M. Losada and N. Rius, 
hep-ph/9911317; M. Bisset, O. C. Kong, C. Macesanu and L. H. Orr, hepph/9811498; M. A. Diaz, E. Torrente-Lujan and J. W. F. Valle, Nucl. Phys. B551, 78 (1999) hep-ph/9808412; M. A. Diaz, J. Ferrandis, J. C. Romao and J. W. F. Valle, Phys. Lett. B453, 263 (1999) hep-ph/9801391. M. A. Diaz, J. Ferrandis, J. C. Romao and J. W. F. Valle, hep-ph/9906343, Nucl. Phys. B, in press A. Akeroyd, M.A. Díaz, J. Ferrandis, M.A. Garcia-Jareño, and J. W. F. Valle, Nucl. Phys. B 529 (1998) 3, hep-ph/9797395. M. Hirsch and J. W. F. Valle, Nucl. Phys. B557, 60 (1999) hep-ph/9812463; M. Hirsch, J. C. Romao and J. W. F. Valle, Phys. Lett. B486 (2000) 255, hep-ph/0002264.

[22] J. W. F. Valle, Phys. Rev. D27 (1983) 1672; J. W. F. Valle and M. Singer, Phys. Rev. D28 (1983) 540.

[23] A. Ioannisian and J. W. F. Valle, hep-ph/9911349.

[24] J. C. Romao, A. Ioannisian and J. W. F. Valle, Phys. Rev. D55, 427 (1997) hep-ph/9607401

[25] I. Antoniadis, Phys. Lett. B246 (1990) 377; N. Arkani-Hamed, S. Dimopoulos, and G, Dvali, Phys. Let. B429, 263 (1998); I. Antoniadis, N. Arkani-Hamed, S. Dimopoulos, and G. Dvali, Phys. Lett. B436, 257 (1998) hep-ph/9804398; N. Arkani-Hamed, S. Dimopoulos, and G, Dvali, Phys. Rev. D59,086004 (1999); K. R. Dienes, E. Dudas, and T. Gherghetta, Phys. Lett. B436,55 (1998); N. Arkani-Hamed, S. Dimopoulos, G, Dvali, and John March-Russell, hepph/9811448; N. Arkani-Hamed, S. Dimopoulos, hep-ph/9811353.

[26] K. R. Dienes and I. Sarcevic, hep-ph/0008144. R. N. Mohapatra, A. PerezLorenzana and C. A. de S Pires, hep-ph/0008158. A. Lukas, P. Ramond, A. Romanino and G. G. Ross, hep-ph/0008049. R. Barbieri, P. Creminelli and A. Strumia, hep-ph/0002199.

[27] M. Apollonio et al., Phys.Lett. B466 (1999) 415, hep-ex/9907037; F. Boehm et al., hep-ex/9912050

[28] J. C. Romao, et al Nucl. Phys. B482 (1996) 3 hep-ph/9604244.

[29] A. Bartl et al, Nucl. Phys. B502 (1997) 19 hep-ph/9612436; A. Bartl, et al hep-ph/0007157; B. Mukhopadhyaya, S. Roy and F. Vissani, Phys. Lett. B443, $191(1998)$

[30] W. Porod, M. Hirsch, J. C. Romao and J. W. F. Valle, in preparation and J.W.F. Valle, talk at the XXXth International Conference on High Energy Physics July 27 - August 2, 2000, Osaka, Japan 